\title{
Preoperative histological classification of primary lung cancer: accuracy of diagnosis and use of the non-small cell category
}

\author{
S L Edwards, C Roberts, M E McKean, J S Cockburn, R R Jeffrey, K M Kerr
}

\begin{abstract}
Aims-To compare the preoperative classification of lung carcinoma made on cytological and histological specimens with the postoperative classification made on the resected specimen. In addition, to find out how often the term "non-small cell lung cancer, not otherwise specified" (NSCLC) was used, and in such cases to note the final diagnosis.
\end{abstract}

Methods-Between 1991 and 1995, 303 patients had a lung resection in Aberdeen for primary carcinoma. For each patient, the departmental records were examined for preoperative specimens (cytological and histological). A note was made of whether each specimen was positive or negative for malignancy and, if positive, what the cell type was. Where patients had more than one sample submitted, the most specific result was taken.

Results-Fifty four per cent of patients had a correct specific preoperative diagnosis of malignancy, whereas $34 \%$ were labelled as NSCLC. Patients with squamous carcinoma were more likely to have a diagnosis of malignancy $(88 \%)$ that was specifically correct $(75 \%)$. Patients who had adenocarcinoma were less likely to have a preoperative diagnosis of malignancy $(64 \%)$ that was specifically correct $(35 \%)$. For those in whom a diagnosis of NSCLC was made, $55 \%$ turned out to have adenocarcinoma whereas $24 \%$ had squamous carcinoma.

Conclusions-By adhering strictly to criteria, a high accuracy of diagnosis can be achieved for squamous carcinoma, but the diagnosis of adenocarcinoma seems to be more of a challenge. NSCLC is a useful and appropriate classification, the use of which reduces the rate of inaccurate specific diagnosis. There are occasions when pathologists can provide a more accurate diagnosis by being less precise. (f Clin Pathol 2000;53:537-540)

Keywords: lung cancer; accuracy of classification; non-small cell

The WHO (1999) classification of lung cancer ${ }^{1}$ is based upon differentiation in the whole tumour, and has no provision for making a non-specific diagnosis that could be refined if further material becomes available. Lung cancers are frequently heterogeneous and biopsy specimens provide only a small amount of tissue from which to make a preoperative diagnosis. Only $10-15 \%$ of patients with lung cancer will have the tumour resected and the preoperative tumour classification confirmed. Therefore, most patients' treatment will be based upon the diagnosis from preoperative specimens alone. In a previous study, ${ }^{2}$ a group of eight pathologists, each with an interest in lung cancer, compared their classification of primary lung cancer on preoperative bronchial biopsy specimens with the agreed diagnosis on the subsequent resected specimen. Their diagnostic accuracy for adenocarcinoma was low $(50 \%)$, and less than that for squamous carcinoma $(75 \%)$. Similar results have been found by others. ${ }^{34}$ It is of concern, therefore, that if $85-90 \%$ of patients have a diagnosis made on preoperative specimens alone, with the likelihood of some degree of inaccuracy in tumour classification from such specimens, then epidemiological studies might be based on flawed data.

Thomas and colleagues ${ }^{2}$ advocated the use of the category "non-small cell carcinoma, not otherwise specified" (NSCLC) in cases where definite features of differentiation were absent, but the lesion lacked features of small cell undifferentiated carcinoma. This increased their diagnostic accuracy by $10-15 \%$. Many centres have now adopted this approach. It has been shown that pathologists are reliable at making the distinction between small cell and other types of lung carcinoma on biopsy specimens, but have more difficulty agreeing among themselves about further classification of those cases that are not small cell carcinoma. ${ }^{5}$ At present there is no need, from a therapeutic perspective, to subclassify NSCLC. With the introduction of neoadjuvant chemotherapy, however, pathologists might well be required to subclassify the non-small cell malignancies because the management protocols might differ for different tumour types.

The aims of our study were to compare the preoperative classification of lung carcinoma made on cytological and histological specimens with the postoperative classification made on the resected specimen, and to determine how often, in a single department, the classification NSCLC was used and, when this occurred, what the final diagnosis was.

\section{Methods}

Between 1991 and 1995, 303 patients had a lung resection in Aberdeen for primary carcinoma. All of these tumours have been reviewed and classified by one of us (KMK), according to WHO criteria, and the information stored 
Table 1 Postoperative tumour classification on resected specimens

\begin{tabular}{lll}
\hline & No. of patients $(n=303)$ & Percentage \\
\hline Adenocarcinoma & 118 & $39 \%$ \\
Squamous carcinoma & 108 & $36 \%$ \\
Adenosquamous carcinoma & 19 & $6 \%$ \\
Large cell undifferentiated carcinoma & 13 & $4 \%$ \\
Neuroendocrine tumours & 13 & $4 \%$ \\
Other tumour types & 32 & $11 \%$ \\
\hline
\end{tabular}

Table 2 Types of preoperative specimen submitted

\begin{tabular}{llll}
\hline & $\begin{array}{l}\text { All patients } \\
(n=237)\end{array}$ & $\begin{array}{l}\text { Patients with squamous } \\
\text { carcinoma }(n=81)\end{array}$ & $\begin{array}{l}\text { Patients with } \\
\text { adenocarcinoma } \\
(n=94)\end{array}$ \\
\hline Cytology specimens only & $49 \%$ & $25 \%$ & $76 \%$ \\
Cytology + biopsy specimens & $46 \%$ & $68 \%$ & $21 \%$ \\
Biopsy specimens only & $4 \%$ & $7 \%$ & $3 \%$ \\
\hline
\end{tabular}

$\star$ Final diagnosis on resected specimens.

on our Grampian Pulmonary Resection Database (GRAMPURD). In each case, multiple blocks of tumour were available for examination. For each patient, the records in the department of pathology, Aberdeen Royal Infirmary were examined for preoperative specimens; namely, sputum, transthoracic fine needle aspirates, and bronchoscopically derived brushings, washings, and biopsies, taken to establish a preoperative diagnosis. It was noted whether each specimen was positive or negative for malignancy and, if positive, the cell type was recorded. This included the designation NSCLC, a classification used in our department since 1990.

Reports of "suspicious" or "atypia" were included with those negative for malignancy. Some patients had more than one type of sample submitted preoperatively. Although each result was recorded, the most specific was taken as the preoperative result because it was the one most likely to have been acted upon clinically. Thus, a report of NSCLC would overwrite any "negative" reports but would itself be overwritten by a specific typed malignant diagnosis on another sample. In those few cases that proved to be adenosquamous carcinoma, a preoperative diagnosis of either adenocarcinoma or squamous carcinoma was accepted as "correct". A diagnosis of large cell undifferentiated carcinoma was regarded as a specific diagnosis.

In our department, bronchial biopsy and all cytology specimens are reported independently by different pathologists. Any instance was noted where there was a discrepancy between specific diagnoses given on biopsy and cytology specimens.

\section{Results}

The starting point of our study was to consider only patients who had resected primary lung carcinoma. Table 1 gives the final tumour classifications on the resected specimens. The low number of neuroendocrine tumours reflects the surgical nature of the series.

Of our 303 patients, 66 had no preoperative specimens on record. Of the remaining 237 patients, 117 patients (49\%) had only cytology specimens sent to our department preoperatively, $110(46 \%)$ had both cytology and biopsy specimens sent, whereas $10(4 \%)$ had only biopsy specimens sent. Of those patients whose final diagnosis was squamous carcinoma, cytology and biopsy specimens were sent in $68 \%$ of cases, whereas in those with adenocarcinoma, cytology only was the most frequent submission (76\%) (table 2).

Of the 237 patients who had specimens submitted to our department, there was no tissue diagnosis of malignancy in 67 (28\%). Overall, 170 patients $(72 \%)$ had a preoperative diagnosis of malignancy. However, preoperative specimens from patients who ultimately proved to have squamous carcinoma gave a "malignancy yield" of $88 \%$, whereas for those with adenocarcinoma it was $64 \%$.

One hundred and seventy patients had preoperative cytology and/or biopsy specimens that showed malignancy. Table 3 shows the comparative data for the group overall and for those with squamous or adenocarcinoma. Whereas the rate of correct specific preoperative diagnosis was $54 \%$ for the group overall, it was better in those with squamous carcinoma $(75 \%)$, but worse in those with adenocarcinoma $(35 \%)$. The frequency of a NSCLC diagnosis was $34 \%$ overall, lower in those with squamous carcinoma (20\%), but higher in those with adenocarcinoma (53\%). An incorrect specific diagnosis was given preoperatively in $11 \%$ of cases. This was an unusual occurrence in squamous carcinoma $(4 \%)$, but more common in patients with adenocarcinoma (10\%). A discrepancy between specific diagnoses given on cytology and biopsy specimens was very uncommon.

Table 4 shows data for bronchial biopsy specimens only. One hundred and twenty patients had bronchial biopsies and in 88 $(73 \%)$ malignancy was diagnosed. The rate of correct specific diagnosis preoperatively was quite high, particularly in those with squamous carcinoma (79\%). Only 14 patients with adenocarcinoma had a malignant preoperative biopsy. Of these, only five were typed correctly, another four were incorrectly typed, and five were classified as NSCLC.

Table 5 shows a similar comparison using only the results from cytology specimens. Of the 227 patients who had cytology specimens

Table 3 Comparison of preoperative and postoperative classification of lung cancer

\begin{tabular}{|c|c|c|c|}
\hline & $\begin{array}{l}\text { All patients } \\
(n=170)\end{array}$ & $\begin{array}{l}\text { Patients with } \\
\text { squamous carcinoma * } \\
(n=71)\end{array}$ & $\begin{array}{l}\text { Patients with } \\
\text { adenocarcinoma * } \\
(n=60)\end{array}$ \\
\hline Correct specific diagnosis given & $54 \%$ & $75 \%$ & $35 \%$ \\
\hline NSCLC & $34 \%$ & $20 \%$ & $53 \%$ \\
\hline Incorrect specific diagnosis given & $11 \%$ & $4 \%$ & $10 \%$ \\
\hline Discrepancy between cytology and biopsy specimen classification & $1 \%$ & $1 \%$ & $2 \%$ \\
\hline
\end{tabular}

*Final diagnosis on resected specimens.

NSCLC, non-small cell lung cancer, not otherwise specified. 
Table 4 Comparison of preoperative tumour classification on bronchial biopsy specimens with postoperative classification

\begin{tabular}{llll}
\hline & $\begin{array}{l}\text { All patients } \\
(n=88)\end{array}$ & $\begin{array}{l}\text { Patients with squamous } \\
\text { carcinoma }(n=53)\end{array}$ & $\begin{array}{l}\text { Patients with } \\
\text { adenocarcinoma } \\
(n=14)\end{array}$ \\
\hline Preoperative classification & $63 \%$ & $79 \%$ & $36 \%$ \\
Correct specific diagnosis given & $24 \%$ & $19 \%$ & $36 \%$ \\
Incorrect specific diagnosis given & $13 \%$ & $2 \%$ & $28 \%$ \\
\hline
\end{tabular}

$\star$ Final postoperative diagnosis.

NSCLC, non-small cell lung cancer, not otherwise specified.

Table 5 Comparison of preoperative tumour classification on cytology specimens with postoperative classification

\begin{tabular}{llll}
\hline & $\begin{array}{l}\text { All patients } \\
(n=138)\end{array}$ & $\begin{array}{l}\text { Patients with squamous } \\
\text { carcinoma }\end{array}(n=48)$ & $\begin{array}{l}\text { Patients with } \\
\text { adenocarcinoma } \\
(n=56)\end{array}$ \\
\hline Preoperative classification & $45 \%$ & $64 \%$ & $32 \%$ \\
Correct specific diagnosis given & $47 \%$ & $29 \%$ & $61 \%$ \\
NSCLC & $8 \%$ & $7 \%$ & $7 \%$ \\
Incorrect specific diagnosis given & $8 \%$ & &
\end{tabular}

ॠFinal postoperative diagnosis.

NSCLC, non-small cell lung cancer, not otherwise specified.

Table 6 Comparison of preoperative diagnosis of squamous carcinoma, adenocarcinoma, and NSCLC with final tumour classification

\begin{tabular}{llll}
\hline & \multicolumn{2}{l}{ Preoperative classification } & \\
\cline { 2 - 4 } Postoperative classification & $\begin{array}{l}\text { Squamous carcinoma } \\
(n=70)\end{array}$ & $\begin{array}{l}\text { Adenocarcinoma } \\
(n=26)\end{array}$ & $\begin{array}{l}\text { NSCLC } \\
(n=58)\end{array}$ \\
\hline Squamous carcinoma & $87 \%$ & $8 \%$ & $24 \%$ \\
Adenocarcinoma & $6 \%$ & $80 \%$ & $55 \%$ \\
Other tumour type & $7 \%$ & $12 \%$ & $21 \%$ \\
\hline
\end{tabular}

NSCLC, non-small cell lung cancer, not otherwise specified.

taken, 138 had a preoperative tissue diagnosis of malignancy by cytology (61\%). Although the "correct specific diagnosis" frequency was lower when compared with biopsy material, the differences between squamous and adenocarcinoma are maintained. NSCLC was a more frequent classification, particularly in adenocarcinoma.

Table 6 shows a comparison of preoperative diagnosis of squamous carcinoma (70 patients), adenocarcinoma (26 patients), and NSCLC (58 patients) with the final outcome in terms of tumour classification. These data show that, when the specific diagnosis of squamous carcinoma or, infrequently, adenocarcinoma was made, the accuracy was relatively high ( $87 \%$ and $80 \%$, respectively). More than half those patients given a diagnosis of NSCLC proved to have adenocarcinoma, whereas around a quarter had squamous carcinoma. None had a neuroendocrine tumour.

\section{Discussion}

The aim of our study was to compare preoperative tumour classification with the final typing of surgical specimens, and examine the usage of the diagnosis NSCLC in the initial investigation and its outcome on surgical resection specimens.

The use of the term non-small cell lung cancer was first suggested by Chuang et al in $1984,{ }^{7}$ but it was little used in the literature until 1993, when Thomas and colleagues ${ }^{2}$ again supported its use. In the latter study, the application of this less specific category improved diagnostic accuracy by $10-15 \%$. Two further studies ${ }^{56}$ attributed to histopathologists an excellent ability to distinguish between small cell carcinoma and NSCLC, and again advocated the use of the term NSCLC, but acknowledged the difficulties in preoperative typing of, in particular, adenocarcinoma, as shown by others. ${ }^{2-6} 8-10$

It is difficult to compare findings from the various published studies with each other and with our own results. Much of the published work concentrates on different methods of cytological preparation, ${ }^{489}$ considers specimens rather than patients, ${ }^{410}$ and might not have a surgical specimen on which to prove the final tissue diagnosis. ${ }^{8}$ There are also differences depending on whether accuracy refers to the number of correct preoperative diagnoses given or the number of occasions the final diagnosis was made correctly preoperatively.

To date, ours is the largest series in the literature, particularly because all cases included had a definitive tumour classification made on a surgical resection specimen. The frequency of submission of cytology and biopsy specimens reflects the presence or absence of visible tumour at bronchoscopy, and the tendency for most squamous carcinomas to be central and most adenocarcinomas peripheral in location.

Our overall yield for malignancy of $72 \%$ compares with $71 \%$ found by Clee et al. ${ }^{10}$ Their yield for cytology specimens was also $71 \%$, although fibreoptic biopsy specimens gave only $58 \%$ malignancy, whereas ours were $61 \%$ and $73 \%$, respectively. Our biopsy figure is nearer to that of Payne et al, ${ }^{3}$ who found a $69 \%$ yield on biopsy specimens; however, they included rigid bronchoscopy specimens, which are generally found to give higher yields. Our cytology specimens include a different range of techniques from those used by Clee et $a l^{10}{ }^{10}$ and cannot account for different practices between bronchoscopists. Truong ${ }^{4}$ reported $66 \%$ sensitivity for malignancy in cytological specimens.

Comparison of accuracy rates is difficult because we used the category NSCLC. All other studies have required allocation of a specific classification in each case. Thus, although our "correct specific diagnosis" rates might appear low in some instances, our rates of "incorrect specific diagnosis" are also very low. Furthermore, our final tumour classification includes very small numbers of large cell undifferentiated carcinoma and a small number of rarities such as carcinosarcoma, mixed tumour (excluding adenosquamous carcinoma), and basaloid carcinoma. Other series seem not to have these unusual tumours and have a higher proportion of both small cell and large cell tumours. Large case numbers of small cell carcinoma will increase overall accuracy because they are recognised as being particularly reliably classified on bronchoscopically derived material. ${ }^{5}$ In general, large cell undifferentiated carcinoma is not a diagnosis that can, or should, be made preoperatively because it requires examination of the whole tumour to exclude evidence of differentiation, especially of adenocarcinoma. The diagnosis of large cell undifferentiated carcinoma was made preoperatively in a small number of patients in our study and almost all of these, and the rare 
tumours in the "others" category (table 1), account for most of our incorrect specific preoperative diagnoses. The same would be true for pleomorphic/spindle cell carcinomas.

There is general agreement that squamous carcinoma and small cell carcinoma have the highest accuracy of diagnosis in small preoperative specimens, ${ }^{348-10}$ whereas adenocarcinoma is less accurately diagnosed. Payne and colleagues $^{3}$ reported only $20 \%$ accuracy, Clee et al reported $50 \%,{ }^{10}$ whereas Truong and colleagues $^{4}$ and Suprun and colleagues ${ }^{8}$ refer to below average or "low" accuracy for adenocarcinoma diagnosis. Thomas et al also reported a figure of $50 \%$ on bronchial biopsy material. ${ }^{2}$ Rather against the trend, Tanaka and colleagues ${ }^{9}$ diagnosed $84 \%$ of their adenocarcinomas correctly. We correctly diagnosed just $35 \%$ of our adenocarcinomas, regardless of specimen type. Overall, however, because of our use of the NSCLC category we gave an incorrect label very infrequently unlike, presumably, most other authors. Although the number of biopsied adenocarcinomas was low, our "incorrect specific" rate was relatively high $(28 \%)$. By trying to give a specific diagnosis when reliable criteria are absent, more errors will be made, particularly with adenocarcinomas. A more frequent use of the NSCLC category will reduce the number of incorrect specific diagnoses, as reflected in our data on cytological diagnosis of adenocarcinoma.

When we made a specific preoperative diagnosis of squamous carcinoma or adenocarcinoma this was, in most cases, correct $(87 \%$ and $80 \%$, respectively). Only Chuang and colleagues $^{7}$ express their data this way and, rather surprisingly, were correct in only $66 \%$ of 32 squamous carcinomas but were correct in $93 \%$ of 44 adenocarcinomas diagnosed on bronchoscopically derived material. Not surprisingly, 21 of their 24 diagnoses of large cell undifferentiated carcinomas proved to be wrong.

Although our rates of correct specific preoperative diagnosis might appear low, this is against a background of very robust postoperative diagnosis using only surgical resection specimens. Included in the series are rare tumour types almost impossible to diagnose preoperatively. Furthermore, the presence of preoperative diagnoses of large cell undifferen- tiated carcinoma will always make accuracy figures worse.

High accuracy of diagnosis can be achieved for squamous carcinoma by adhering strictly to criteria for squamous differentiation (keratin, intercellular bridges). Diagnosis of adenocarcinoma seems to be more of a challenge. Mucin and glands, if present, permit the diagnosis but are frequently not present in bronchial biopsy or cytological material and, as Thomas and colleagues $^{2}$ pointed out, other "so called" adenocarcinoma criteria are very unreliable. Thus, we agree that NSCLC is a useful and more appropriate classification to use, reflecting the view that it is sometimes better to be more accurate by being less precise. We found it provided the best classification that we could give in $34 \%$ of our patients and that, when used, $55 \%$ of patients would have an adenocarcinoma, while $24 \%$ would eventually prove to have squamous carcinoma. We shall continue to use this classification and support moves to account for this category in disease coding systems for registering diagnoses of lung cancer. Epidemiological data will be more useful if based upon more robust histological diagnosis.

1 Travis WD, Colby TV, Corrin B, et al. World Health Organisation. Histological typing of lung and pleural tumours, 3rd ed. International histological classification of tumours, Vol 1. Berlin: Springer Verlag, 1999

2 Thomas JStJ, Lamb D, Ashcroft T, et al. How reliable is the diagnosis of lung cancer using small biopsy specimens? Report of a UKCCCR lung cancer working party. Thorax 1993;48:1135-9.

3 Payne CR, Stovin PGI, Barker V, et al. Diagnostic accuracy of cytology and biopsy in primary bronchial carcinoma. Thorax 1979;34:294-9.

4 Truong LD, Underwood RD, Greenberg SD, et al. Diagnois and typing of lung carcinomas by cytopathologic methods. A review of 108 cases. Acta Cytol 1985;29:379-84.

5 Burnett RA, Swanson Beck J, Howatson SR, et al. Observer variability in histopathological reporting of malignant bronchial biopsy specimens. F Clin Pathol 1994;47:711-13.

6 Burnett RA, Howatson SR, Lang S, et al. Observer variability in histopathological reporting of non-small cell lung carcinoma on bronchial biopsy specimens. $f$ Clin Pathol 1996;49:130-3.

7 Chuang MT, Marchevsky A, Teirstein AS, et al. Diagnosis of lung cancer by fibreoptic bronchoscopy: problems in the histological classification of non-small cell carcinomas. Thorax 1984;39:175-8.

8 Suprun H, Pedio G, Ruttner JR. The diagnostic reliability of cytologic typing in primary lung cancer with a review of the

9 Tanaka T, Yamamoto M, Tamura T, et al. Cytological and histological correlation of primary lung cancer: a preliminary study of 106 cases with resectable tumours. Acta Med Okayama 1983;37:11-19.

10 Clee MD, Duguid HLD, Sinclair DJM. Accuracy of morphological diagnosis of lung cancer in a department of respiratory medicine. 7 Clin Pathol 1982;35:414-19. 